\title{
Medicina de Familia y Comunidad y su papel en evitar los excesos sanitarios (preventivos, diagnósticos, terapéuticos y rehabilitadores)
}

\author{
Family and Community Medicine and its role in preventing health \\ overuse (preventive, diagnostic, therapeutic and rehabilitative)
}

Juan Gérvas (https://orcid.org/0000-0002-7802-5457)

Lourdes Luzón Oliver (https://orcid.org/0000-0001-5401-115X) ${ }^{2}$

Mercedes Pérez-Fernandez (https://orcid.org/0000-0002-9285-1739) ${ }^{1}$

\footnotetext{
${ }^{1}$ Equipo CESCA. Pradillo 68. 28002 Madrid España. jjgervas@gmail.com ${ }^{2}$ Centro de Salud de Navamorcuende. Toledo

España.
}

\begin{abstract}
In Medicine, it is critical "to offer 100\% of what is needed and avoid 100\% of what is not needed." Unfortunately, this primary issue is challenging, and generally, more than required is offered, and everything that is unnecessary is not avoided. This is a nonsystematic review with a teaching objective that reviews the general issue in primary care and suggests ways to avoid overuse and shortcomings concerning preventive, diagnostic, therapeutic, and rehabilitative interventions. Knowing not to do is science and art that is hardly taught and practiced less. The overuse that harm are an almost daily part of clinical practice in prevention, diagnosis, treatment, and rehabilitation. It is essential to promote "the art and science of not doing".
\end{abstract}

Key words Primary care, Medical overuse, Unnecessary procedures
Resumen En Medicina es clave "ofrecer el 100\% de lo que se precisa y evitar el 100\% de lo que no se precisa”. Lamentablemente, es difícil cumplir con esta cuestión clave y generalmente se ofrece más de lo que se precisa y no se evita todo lo que no se precisa. Este texto es una revisión no sistemática con objetivo docente en que se revisa el problema general en atención primaria y se proponen formas de evitar los excesos y defectos respecto a intervenciones preventivas, diagnósticas, terapéuticas y de rehabilitación. Saber no hacer es ciencia y arte que se enseña poco y se practica menos. Los excesos que dañan son parte casi diaria de la práctica clínica, en prevención, diagnóstico, tratamiento y rehabilitación. Es imprescindible fomentar "el arte y la ciencia de no hacer".

Palabras clave Atención Primaria, Sobreuso médico, Intervenciones innecesarias 


\section{Introducción}

La salud y el bienestar no dependen de los médicos, salvo en situaciones muy específicas. Lo fundamental es la educación que imparten los maestros y el suministro y depuración de agua, además del desarrollo económico, la justa distribución de la riqueza y el acceso a una vivienda y un trabajo digno. Se ha calculado que la mejora de la expectativa de vida se debe en un $10 \%$ al sistema sanitario, y en el $90 \%$ a otros determinantes de salud ${ }^{1}$.

Visto con perspectiva histórica, la Medicina ofrece lo que pueden parecer milagros laicos. Por ejemplo, el consejo contra el tabaco que logra el abandono del mismo por el paciente, la anestesia subcutánea que permite extirpar un cáncer de piel sin dolor, la vacuna contra la rabia que evita un terrible proceso tras la infección, la recomendación del consumo de sal iodada que evita el hipotiroidismo, el uso de penicilina en una neumonía, etc. Son ejemplos de una Medicina que espera la población, y que por ello le parece lo mínimo y exige "más y más" Medicina, como si más fuera equivalente a mejor y como si todo fuera evitable.

Los médicos no tenemos la misión de evitar todo sufrimiento y muerte. Nuestro cometido es más modesto y simple: disminuir la morbilidad y mortalidad innecesariamente prematura y sanitariamente evitable (MIPSE) ${ }^{2}$. Es decir, trabajar de forma que evitemos el sufrimiento y la muerte que pueden disminuir con nuestra actividad. Es ejemplo de MIPSE el sufrimiento y la mortalidad por tétanos, pues podemos vacunar y evitarlo.

En Medicina es clave "ofrecer el 100\% de lo que se precisa y evitar el $100 \%$ de lo que no se precisa". Es decir, hay que conseguir el delicado encaje de ofrecer justo lo que se precisa, y sólo eso.

El buen médico sabe lo que tiene que hacer y cuándo y, sobre todo, debería saber lo que no tiene que hacer. Pero es más fácil saber qué hacer y cuándo que saber qué no hacer. Como dijo un cirujano: "Hacen falta 3 meses para aprender a hacer una operación, 3 años para saber cuándo hacerla y 30 años para saber cuándo no hacerla"3.

En este texto hacemos una revisión no sistemática con objetivo docente analizando excesos $y$ defectos de actividades frecuentes en prevención, diagnóstico, tratamiento y rehabilitación en atención primaria.

\section{La longitudinalidad en Atención Primaria y "el arte y la ciencia de no hacer"}

Es longitudinalidad la relación que se establece a largo plazo entre el médico general/de familia y los pacientes de su consulta. Se define como: a) la atención por el mismo médico a lo largo de toda la vida de la mayoría de los problemas del paciente y b) el reconocimiento de la población y de los pacientes de una fuente de cuidados con la cual se cuenta para el contacto inicial y para el seguimiento de los problemas ${ }^{4}$.

La longitudinalidad es clave para contar con la confianza de pacientes, familias y comunidades pues, cuando existe, el médico es el "responsable" del paciente y puede ir corrigiendo sus decisiones según pasa el tiempo. Es decir, el médico es libre para graduar su respuesta según la evolución del cuadro clínico. La longitudinalidad permite que el médico diga al paciente "Parece un catarro, no hace falta hacer nada especial sólo remedios caseros. Pero si algo va mal, sube y dura mucho la fiebre, hay dificultad para respirar o cualquier cosa que le preocupe, no dude en tomar contacto conmigo".

El paciente acepta la incertidumbre en la confianza de que "su" médico corregirá la decisión si hay razones para ello, y el médico puede dominar su incertidumbre clínica en la confianza de que el paciente retornará si el cuadro no evoluciona como estaba previsto. Se evita así el exceso en la respuesta al contar con la ayuda de la evolución en el tiempo; técnicamente es lo que denominamos "esperar y ver".

La longitudinalidad permite la práctica profesional de médicos que son comedidos en sus intervenciones, con formación suficiente para ser capaces de "esperar y ver" y de manejar la "espera expectante" (cuando no hacer nada y esperar es la mejor opción diagnóstica y terapéutica).

Es lo que en inglés se denomina "deliberate clinical inertia" (inercia clínica intencionada), el arte y la ciencia de no hacer nada como una opción lógica y prudente tan típica del buen médico de general/de familia que ni siquiera precisa "diagnosticar-etiquetar" para resolver un alto porcentaje de problemas de salud pero que también sirve en campos tan dispares como urgencias y psiquiatría ${ }^{5-7}$.

En Atención Primaria, la longitudinalidad da una red de seguridad que permite la práctica prudente y sensata del "esperar y ver", con la consiguiente disminución en el uso innecesario de recursos. En países muy desiguales, como Brasil, la longitudinalidad permite adecuar la oferta 
a la necesidad, por el conocimiento profundo de pacientes, familias y comunidades a las que se atiende. También facilita el uso adecuado de los recursos, ya que la longitudinalidad permite la toma juiciosa de decisiones con importante eficiencia económica por el acúmulo de información previa.

\section{¿Cómo evitar los excesos preventivos?}

Lo clave para evitar los excesos preventivos en Atención Primaria es lograr que el médico general/de familia tenga claro que la prevención no es una característica básica de la atención primaria. "La prevención no es una función de la atención primaria sino una actividad complementaria y necesaria para el cumplimiento de sus fines clínicos"s.

En relación con la prevención en Atención Primaria, la verdadera ventaja es que el médico conoce a fondo a sus pacientes, familias y comunidades de forma que puede ofrecerles exactamente lo que necesitan adaptándolo a sus creencias, expectativas y cultura. Se evitan así, por ejemplo, los chequeos, esos reconocimientos rutinarios que carecen de fundamento científico ${ }^{9}, \mathrm{o}$ los cribados (screening) sin fundamento ${ }^{10}$.

Las intervenciones médicas son cada vez más poderosas, más precoces, más variadas y aplicadas por más profesionales distintos y su imagen final, como hemos señalado, parece omnipotente en el sentido de que todo se puede evitar.

El sufrimiento, la enfermedad y la muerte parece que se deben a fallos de la prevención ${ }^{11}$. Por ello es clave establecer un nuevo compromiso con la sociedad y los pacientes para ofrecer sólo lo que "vale la pena" (el 100\% de lo que se precisa y nada del $100 \%$ que no se precisa) en el caso considerado y para la sociedad y ejercer una Medicina basada en la ética de la negativa y la ética de la ignorancia.

Trabajar con la ética de la negativa supone decir "no" de forma apropiada y justificada, con suavidad y cortesía, ante las solicitudes excesivas de pacientes y familiares, compañeros y superiores $^{12}$. La ética de la negativa exige un enorme profesionalismo, un fuerte compromiso con la profesión y con los enfermos, y un caudal inagotable de conocimiento científico.

Trabajar con la ética de la ignorancia supone decir con franqueza y oportunamente "no lo sé”, "no lo sabemos", "no hay conocimiento científico al respecto"13. Es decir, supone compartir con pacientes y familiares, compañeros y superiores los límites de la Ciencia y de la Medicina.
Cuanto más sabemos, más conscientes somos de nuestra ignorancia. Cuanto más ignorantes, más arrogantes e imprudentes. Conviene refrenar las expectativas excesivas que en prevención son casi infinitas, a veces alimentadas por una Medicina que promete imposibles, casi la juventud eterna; por ejemplo, con la Medicina de Precisión y el "Big Data"14.

Exceso preventivo frecuente, muchas veces estimulado por incentivos institucionales y guías de práctica clínica, es el uso de tablas de riesgo como tablas de decisión. Por ejemplo, no existe conocimiento científico que sustente el uso de las tablas de riesgo cardiovascular como tablas de decisión, pues carecen de "análisis clínico de impacto” (el impacto de su aplicación en la mejora de la salud de los pacientes). Es decir, las tablas de riesgo no permiten predecir quién tendrá más probabilidad de tener un evento cardiovascular.

El médico clínico transforma erróneamente las tablas de riesgo cardiovascular en tablas de decisión por más que se haya demostrado que evaluar el riesgo cardiovascular no lleva a mejor salud de los pacientes ${ }^{15}$.

Por último, para evitar los excesos en la prevención hay que tener en cuenta, contra la creencia popular, que no siempre es mejor prevenir que curar ${ }^{16}$ y por ello conviene la mirada precavida ante las propuestas preventivas, muchas veces basadas en la falacia de Beveridge (creer que la prevención disminuye los costes $)^{17}$.

\section{¿Cómo evitar los excesos diagnósticos?}

Es un gran error el seguir mantras tan simples como «en la duda, pide pruebas», o «en la duda, al menos etiqueta». A los estudiantes y residentes de ciencias de la salud, y a los médicos, se nos enseña, teórica y prácticamente, que es siempre preferible hacer de más que de menos, en el buen supuesto de que la intervención médica tiene escasos riesgos, o ninguno. Además, se nos impone el diagnóstico como barrera para la toma de decisiones terapéuticas, de forma que se enseña a trabajar con "la tiranía del diagnóstico"18.

La realización de pruebas es la norma, con baterías cuya mayor dificultad es la interpretación de resultados anormales inesperados; así, por ejemplo, quedan por aclarar y explicar entre el 30 y el $60 \%$ de los resultados anormales encontrados en las pruebas habituales de los protocolos preoperatorios (que, además, generalmente carecen de fundamento científico $)^{19}$.

También se sabe que la realización de pruebas diagnósticas no tranquiliza al paciente. En pa- 
cientes preocupados, en los que el médico tiene escasas dudas acerca de la necesidad de pruebas varias $^{20}$, la realización de dichas pruebas, y su normalidad, no calma ni la preocupación ni la ansiedad de los pacientes.

Resulta inútil el pedir pruebas diagnósticas cuando es baja la probabilidad de que sean anormales. Estas pruebas ni tranquilizan a los pacientes, ni disminuyen su ansiedad ni ayudan a que se resuelvan sus síntomas.

En muchos casos, además, los resultados anormales por pruebas innecesarias provocan una "cascada" de intervenciones para aclararlas, que pueden acabar muy mal. Ese fue el caso de un paciente anciano al que su familia le regaló un "reloj inteligente" para controlar el pulso ${ }^{21}$. Llevando una vida plena independiente de jubilado, el reloj demostró una mínima anomalía del ritmo, que acabó en la realización de una coronariografía, que movió una placa arterial, que provocó un ictus cerebral, que llevó al paciente a la reclusión en un asilo, y en silla de ruedas. Este caso es ejemplo del "efecto cascada" en el que un hallazgo irrelevante lleva a un daño importante.

En el caso de las resonancias magnéticas (RMN) y tomografías computarizadas (TACs) se producen hallazgos casuales, "incidentalomas", hasta en el $38 \%$ de los casos, que luego hay que estudiar con las cascadas consiguientes ${ }^{22}$. En otro ejemplo, para hacernos idea de la frecuencia del efecto cascada tras solicitar pruebas innecesarias, se dio en el 16\% de los pacientes con electrocardiograma pre-operatorio en cirugía de catara$\operatorname{tas}^{23}$. En un estudio canadiense, comparando con los pacientes de bajo riesgo que no se sometieron a un ECG, los que sí lo hicieron tuvieron mayores probabilidades de ser sometidos a más pruebas posteriormente: pruebas de esfuerzo (6,5 veces más probable), ecocardiogramas ( 7,1 veces más probable) y derivación a especialista de cardiología $(5,4$ veces más probable) y todo esto, sin necesidad porque no hubo eventos cardiovasculares significativos ${ }^{24}$.

Así mismo, se ha demostrado el sobreuso de métodos diagnósticos con imagen para pacientes con dolor de espalda inespecífico, sin signos de alarma, lo que además de aumentar los costes expone al paciente a daños innecesarios como irradiación y cascadas terapéuticas. Pero pacientes y profesionales tienden a infraestimar el daño ocasionado por el exceso de pruebas preventivas, diagnósticas y terapéuticas ${ }^{25,26}$.

Conviene enseñar a estudiantes, residentes, clínicos y pacientes sobre el efecto cascada en la atención clínica. Dichas cascadas (iniciadas por signos y síntomas irrelevantes, por actividades preventivas o por decisiones diagnósticas-terapéuticas erróneas generadas por guías y protocolos, agreden al paciente) ignoran la yatrogenia implícita en todo acto médico y disminuyen la eficacia de la actividad profesional, al diluir el esfuerzo de los médicos sobre enfermos y enfermedades de muy distinta gravedad ${ }^{27,28}$.

\section{¿Cómo evitar los excesos terapéuticos?}

Lo clave ante la intervención terapéutica es tener en cuenta que toda recomendación médica puede tener efectos adversos. Sólo se justifica la intervención médica cuando es muy probable que sea positivo el balance entre beneficios y daños.

La actitud intervencionista que favorece la aceptación preferente de los errores por actividades en exceso no es nada inocente y conduce, por ejemplo, a que millones de pacientes sean etiquetados en falso y tratados como hipertensos, con la morbilidad y mortalidad que comportan el simple erróneo etiquetado de enfermo y los medicamentos contra la hipertensión ${ }^{29}$.

En otro ejemplo, el uso innecesario de suplementos de vitamina $\mathrm{D}$, se mezclan los excesos en el diagnóstico (determinación plasmática) con los del terapéutica (la administración de suplementos). "El aporte exógeno de vitamina D no proporciona beneficio clínico en mortalidad, enfermedades cardiovasculares, metabólicas ni cáncer" ${ }^{\prime 3}$. El aporte médico de vitamina D puede dar lugar a toxicidad con grave hipercalcemia, lo que llevó a lanzar una alarma a la Agencia Española del Medicamento: "Vitamina D: casos graves de hipercalcemia por sobredosificación en pacientes adultos y en pediatría"31.

El sobreuso de antibióticos provoca problemas adicionales, por la externalidad negativa que conlleva el establecimiento de resistencias bacterianas, de forma que el daño no es sólo individual, sino de terceros, de la sociedad en conjunto. Los antibióticos no se precisan, en general, ni en las otitis, ni en las sinusitis, ni en las faringitis, ni en las bronquitis, ni en las cistitis, ni (quizá) en las amigdalitis.

Tampoco se precisan antibióticos en la profilaxis de las endocarditis, pues su uso provoca más daños que beneficios. El uso y abuso de los antibióticos es un problema de salud pública, con externalidad negativa tal que provoca miles de muertos anuales ${ }^{32}$.

Para terminar con los ejemplos de excesos en el tratamiento, nada como el "encarnizamiento" con los diabéticos y los objetivos imposibles en 
el control glucémico, especiamente los niveles de hemoglobina glucosilada ${ }^{33}$. Los niveles de $\mathrm{Hgb}$ A1C deberían ser lo suficientemente bajos como para disminuir los síntomas, pero no tanto como para correr el riesgo de hipoglucemia. Para muchos pacientes, $8 \%$ a $9 \%$, con un nivel de glucosa en sangre en ayunas de menos de $200 \mathrm{mg} / \mathrm{dl}^{34}$. Lamentablemente, muchos sistemas de incentivos y guías clínicas proponen justo el control glucémico agresivo.

El problema es que muchas veces los excesos forman parte del "estado del conocimiento" e incluso son forzados por guías y protocolos y por sistemas de incentivos. La actitud profesional complaciente y cómoda de aceptar las pautas habituales y establecidas, de errar comúnmente por exceso, rompe por completo el compromiso ético de actuar como agente del paciente, lo que implica decidir como decidiría éste si tuviese capacidad para obrar con un juicio clínico correctamente formado.

\section{¿Cómo evitar los excesos rehabilitadores?}

Entre el 50 y el 70\% de la población presentará algún problema osteomuscular a lo largo de su vida y entre ellos los más frecuentes son los dolores de cuello, lumbares y de hombro ${ }^{35}$. A tener en cuenta que la mayoría de estos problemas de salud están relacionados con condiciones de trabajo y socioeconómicas.

Existe gran variabilidad de práctica clínica en cuanto a los tratamientos rehabilitadores prescritos; así para pacientes con características similares los tratamientos son diferentes en función de qué profesional le haya atendido primero (reumatólogo, rehabilitador, médico de familia, fisioterapeuta, etc.), la cartera de servicios ofertada en la región donde resida y la disponibilidad y lista de espera de dichos tratamientos.

Algunos estudios como el de Elshaug et al. ${ }^{36}$ listaron 150 de prácticas en el cuidado de salud de poco valor (prácticas que generarían poco valor o que causarían daño). Ejemplo de práctica de poco valor es las artroscopia, de las que en Australia se realizaron 33.682 a pesar de que ensayos clínicos aleatorizados han demostrado su poca efectividad en relación a placebo en pacientes con desgarro de menisco ${ }^{37}$.

El dolor de espalda es una de las causas más frecuentes de consulta en el mundo y existe demostración del poco valor de las prácticas utilizadas en su manejo ${ }^{38}$. Por ejemplo, en España se demostró que el $60 \%$ de los recursos gastados en el tratamiento del dolor de cuello, espalda y hom- bro lo habían sido en tratamientos para los que no existían pruebas de efectividad.

Una revisión sistemática demostró la mejora del dolor lumbar inespecífico cuando se realizan intervenciones educativas en Atención Primaria con un excelente NNT (número necesario de pacientes a tratar) de 17 , siendo los resultados mejores cuando las intervenciones son realizadas por médicos de familia que por otros profesionales (enfermería o fisioterapia) ${ }^{39}$.

Otro ensayo clínico ${ }^{40}$ en Atención Primaria demostró que en pacientes con dolor lumbar los programas de "educación" en grupo mejoraban la capacidad funcional, la calidad de vida y los pensamientos catastróficos. En ancianos institucionalizados se ha demostrado que la educación sobre el manejo activo es efectiva para mejorar la restricción de las actividades cotidianas debidas a dolor lumbar, tanto en los sujetos que lo padecen como en aquellos sin dolor, siendo más efectiva que la educación sobre higiene postural ${ }^{41}$. Al estudiar la efectividad y coste/efectividad de la GDS (Godelieve Denys-Struyf) para el tratamiento de la lumbalgia en Atención Primaria observaron que en el grupo "GDS colectivo" el grado de incapacidad mejoró ligeramente más que en el grupo "control", y en el grupo individual ${ }^{42}$. Se trata de intervenciones sencillas, en grupo, y de bajo coste y sin daños para el paciente.

La cuestión pasa por dejar de hacer para poder hacer, y financiar lo que ha demostrado ser más coste/efectivo. Mucho de lo que se hace no sigue las evidencias científicas ${ }^{43}$. No existe fundamento científico para usar los ultrasonidos ni las ondas de choque para el tratamiento del dolor lumbar; no hay pruebas científicas válidas de que sean mejor que el placebo ${ }^{44}$, así como tampoco lo hay para la IDET (electrotermocoagulación intradiscal), que además conlleva riesgos para los pacientes $^{45}$.

La Asociación Australiana de Fisioterapia listó seis prácticas que deberían de dejar de realizarse por el poco valor que aportan. Entre ellas estarían la solicitud de radiografías sin indicación clara, electroterapia en dolor lumbar, o las maniobras de presión en la capsulitis adhesiva del hombro.

En 2014 The American Physical Therapy Association publicó una lista de cinco prácticas rehabilitadoras de bajo valor. Entre estas prácticas se incluían yacuzzis para el manejo de heridas, calor profundo o superficial para mejorar las condiciones musculares, y aparatos que ejerzan movimientos continuos pasivos en pacientes con prótesis de rodilla ${ }^{34}$. 


\section{Conclusión}

Entre las causas de las prácticas de bajo valor que llevan al sobrediagnóstico y al sobretratamiento se incluyen la disposición de tecnología y factores culturales, profesionales y comerciales. Por ejemplo, el disponer de tecnologías con mayor sensibilidad hace que se diagnostiquen y se traten imágenes que pueden estar presentes en gran parte de la población asintomática. De esta forma se "etiqueta" al paciente y se le recomienda un tratamiento y seguimiento definido (lo que supone, a veces, el acceso a ciertos derechos so-

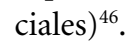

Tanto profesionales como pacientes tienen dificultad para enfrentarse a la incertidumbre e inespecificidad de los síntomas y la respuesta puede ser muy diferente en función del lugar del sistema sanitario donde se inicie el proceso ${ }^{47}$. El miedo y la preocupación por la enfermedad llevan al paciente a consultar más y a someterse a mayor número de pruebas y tratamientos con la creencia ciega en la tecnología y la Medicina y con el pensamiento de que es mejor cuanto más pruebas y cuanto antes se hagan, sin tener en cuenta los daños. Miedo de los pacientes a no ser comprendidos por su médicos, miedo de los médicos al fracaso y/o a ser demandados, miedo a la propia incertidumbre que paraliza e impide ejercer con libertad ${ }^{48}$. El cuidado innecesario sustituye al necesario sobre todo cuando el segundo está menos remunerado ${ }^{49}$.

Habría que adecuar incentivos a calidad y resultados de las prácticas porque al final todos estos problemas tienen un impacto altísimo en la sociedad, en el sistema sanitario y en el paciente $\mathrm{e}^{50,51}$. Saber no hacer es ciencia y arte que se enseña poco y se practica menos. Los excesos que dañan son parte casi diaria de la práctica clínica, en prevención, diagnóstico, tratamiento y rehabilitación. Es imprescindible fomentar "el arte y la ciencia de no hacer".

\section{Colaboradores}

J Gérvas, LL Oliver y M Pérez-Fernandez trabajaron juntos para producir el manuscrito. 


\section{Referencias}

1. Kaplan RM, Milstein A . Contributions of health care to longevity: a review of 4 estimation methods. Ann Fam Med 2019; 17(3):267-272.

2. Ortún V, Gispert R. Exploración de la mortalidad prematura como guía de política sanitaria e indicador de calidad asistencial. Med Clin (Barc) 1988; 90:399403.

3. Gil I. "La verdad es aterradora": los secretos de quirófano según el neurocirujano británico Henry Marsh. BBC Mundo, Salud 24 enero 2017. [accesado en 2019 Jun 8]. Disponíble en: https://www.bbc.com/mundo/ noticias-38731223

4. Gérvas J, Fernández MP, Sánchez RJS. Longitudinalidad, prestigio, buena reputación (social y profesional) y medicina general/de familia. Aspectos clínicos y de salud pública. Informe SESPAS 2012; 26(S1):52-56.

5. Ortiz Lobo A. El arte de hacer el mínimo daño en salud mental. 8 octubre 2016. [accesado en 2019 Jun 8]. Disponíble en: http://www.nogracias.eu/2016/10/08/ el-arte-de-hacer-el-minimo-dano-en-salud-mentalpor-alberto-ortiz-lobo/

6. Keijzers G, Cullen L, Egerton-Warburton D, Fatovich DM. Don't just do something, stand there! The value and art of deliberate clinical inertia. Emerg Med Australas 2018; 30(2):273-278.

7. Gérvas J. El arte y la ciencia de "no hacer nada" (inercia clínica intencionada). [accesado en 2019 Jun 8] Disponíble en: https://www.actasanitaria.com/el-arte-y-la-ciencia-de-no-hacer-nada-inercia-clinica-intencionada/

8. Starfield B, Gérvas J. Prevención de la población al paciente y viceversa. ¿Es la prevención una función de la atención primaria? El Médico 2009; 1104 (Noviembre):34-36.

9. Heneghan C, Mahtani KR. Is it time to end general health checks? BMJ Evid Based Med 2019; pii: bmjebm-2019-111227.

10. Gérvas J, Pérez-Fernández M. Cribados: una propuesta de racionalización. Gac Sanit 2013; 27:372-373.

11. Fugelli P. The Zero-vision: potential side effects of communicating health perfection and zero risk. $\mathrm{Pa}$ tient Educ Couns 2006; 60(3):267-271.

12. Gérvas J. Pérez Fernández M. Uso y abuso del poder médico para definir enfermedad y factor de riesgo, en relación con la prevención cuaternaria. Gac Sanit 2006; 20(Supl. 3):66-71.

13. Smith R. The ethics of ignorance. J Med Ethics 1992; 18(3):117-118, 134

14. Vogt H, Green S, Ekstrøm CT, Brodersen J. How precision medicine and screening with big data could increase overdiagnosis. BMJ 2019; 366:15270.

15. Collins DR, Tompson AC, Onakpoya IJ, Roberts N, Ward AM, Heneghan CJ. Global cardiovascular risk assessment in the primary prevention of cardiovascular disease in adults: systematic review of systematic reviews. BMJ Open 2017; 7(3):e013650.

16. Gérvas J, Starfield B, Heath I. Is clinical prevention better than cure? Lancet 2008; 372:1997-1999 [accesado en 2019 Jun 8]. Disponíble en: http://equipocesca. org/is-clinical-prevention-better-than-cure/

17. Heath I. Prevention in the medical encounter. [accesado en 2019 Jun 8]. Disponíble en: https://docs.google. com/document/d/1F9DLqvzbYoelni-IH7sbfx4Wcqmd8UQgS5xiHxsS3Pg/edit?pli=1
18. Rosenberg CE. The tyranny of diagnosis: specific entities and individual experience. Milbank Q 2002; 80(2):237-260.

19. Roizen MF. More preoperative assessment by physicians and less by laboratory tests. $N$ Engl J Med 2000; 342(3):204-205.

20. Rolfe A, Burton C. Reassurance after diagnostic testing with a low pretest probability of serious disease: systematic review and meta-analysis. JAMA Intern Med 2013; 173(6):407-416.

21. Mandrola J. A contrarian view of digital health. May 17, 2019 [accesado en 2019 Jun 8]. Disponíble en: https://quillette.com/2019/05/17/a-contrarian-viewof-digital-health/

22. Morgan DJ, Dhruva SS, Coon ER, Wright SM, Korenstein D. 2019 Update on Medical Overuse: A Review. JAMA Intern Med 2019.

23. Ganguli I, Lupo C, Mainor AJ, Raymond S, Wang Q, Orav EJ, Chang CH, Morden NE, Rosenthal MB, Colla $\mathrm{CH}$, Sequist TD. Prevalence and Cost of Care Cascades After Low-Value Preoperative Electrocardiogram for Cataract Surgery in Fee-for-Service Medicare Beneficiaries. JAMA Intern Med 2019.

24. Bhatia RS, Bouck Z, Ivers NM, Mecredy G, Singh J, Pendrith C, Ko DT, Martin D, Wijeysundera HC, Tu JV, Wilson L, Wintemute K, Dorian P, Tepper J, Austin PC, Glazier RH, Levinson W. Electrocardiograms in Low-Risk Patients Undergoing an Annual Health Examination. JAMA Intern Med 2017; 177(9):13261333.

25. Hoffmann TC. Patients' expectations of the benefits and harms of treatments, screening, and tests a systematic review. JAMA Intern Med 2015; 175(2):274286.

26. Hoffmann TC, Del Mar C. Clinicians' Expectations of the Benefits and Harms of Treatments, Screening, and Tests: A Systematic Review. JAMA Intern Med 2017; 177(3):407-419.

27. Mold JW, Stein HF. The cascade effect in the clinical care of patients. N Engl J Med 1986; 314(8):512-514

28. Pérez Fernández M, Gérvas J. El efecto cascada: implicaciones clínicas, epidemiológicas y éticas. Med Clin (Barc) 2002; 118(2):65-67.

29. Bell KJL, Doust J, Glasziou P. Incremental Benefits and Harms of the 2017 American College of Cardiology/American Heart Association High Blood Pressure Guideline. JAMA Intern Med 2018; 178(6):755-757.

30. Leache L, Saíz LC, Erviti J. Cribado y suplementación de Vitamina D en adultos. Sol y sombras. BIT 2019; 26(4).

31. Agencia Española de Medicamentos y Productos Sanitarios (AEMPS). Vitamina D: casos graves de hipercalcemia por sobredosificación en pacientes adultos y en pediatría. 19 marzo 2019. [accesado en 2019 Jun 8] Disponíble en: https://www.aemps.gob.es/informa/ notasInformativas/medicamentosUsoHumano/seguridad/2019/NI_MUH_FV-2-2019-vitamina-D.htm

32. Gérvas J. La resistencia a los antibióticos, un problema de salud pública. Aten Primaria 2000; 25(8):589-596. 
33. Kähler P, Grevstad B, Almdal T, Gluud C, Wetterslev J, Lund SS, Vaag A, Hemmingsen B. Targeting intensive versus conventional glycaemic control for type 1 diabetes mellitus: a systematic review with meta-analyses and trial sequential analyses of randomised clinical trials. BMJ Open 2014; 4(8):e004806.

34. Anónimo. ¡Diabético, espabile! Es muy posible que su médico no se entere. Lea esto y enséñeselo. 28 julio 2015. [accesado en 2019 Jun 8]. Disponíble en: http:// www.nogracias.eu/2015/07/28/si-usted-es-diabeticoy-su-medico-quiere-bajarle-el-azucar-por-debajode-200-ensenele-este-texto/

35. Serrano-Aguilar P, Kovacs F, Cabrera-Hernández J, Ramos-Gõi J, García-Pérez L. Avoidable costs of physical treatments for chronic back, neck and shoulder pain within the Spanish National Health Service: A cross-sectional study. BMC Musculoskelet Disord 2011; 12:287.

36. Elshaug AG, Watt AM, Mundy L, Willis CD. Over 150 potentially low-value health care practices: an Australian study. Med J Aust 2012; 197(10):556-560.

37. Thorlund JB, Juhl CB, Roos EM, Lohmander LS. Arthroscopic surgery for degenerative knee: systematic review and meta-analysis of benefits and harms. $B M J$ 2015; 350:h2747

38. Traeger AC, Moynihan RN, Maher CG. Wise choices: making physiotherapy care more valuable. J Physiother 2017; 63(2):63-65.

39. Traeger AC, Hübscher M, Henschke N, Moseley GL, Lee H, McAuley JH. Effect of primary care based education on reassurance in patients with acute low back pain systematic review and meta-analysis. JAMA Intern Med 2015; 175(5):733-743.

40. Albaladejo C, Kovacs FM, Royuela A, Del Pino R, Zamora J. The efficacy of a short education program and a short physiotherapy program for treating low back pain in primary care: A cluster randomized trial. Spine (Phila Pa 1976) 2010; 35(5):483-496.

41. Kovacs F, Abraira V, Santos S, Díaz E, Gestoso M, Muriel A, Gil del Real MT, Mufraggi N, Noguera J, Zamora J; Spanish Back Pain Research Network. A comparison of two short education programs for improving low back pain-related disability in the elderly: A cluster randomized controlled trial. Spine (Phila Pa 1976) 2007; 32(10):1053-1059.

42. Diaz-Arribas MJ, Kovacs FM, Royuela A, Ramos-sa M, Fernández-Serrano M, Gutiérrez-Fernández L, San Martín-Pariente O, Abraira V, Ramos-Sánchez M, Llorca-Palomera R, Pardo-Hervás P, Gestoso M, Sánchez-Gil GC, Elena-Lucas MA, Paniagua-de-la-Calle R, Castellanos-López I, García-Heredia MA, CerónSanz AM, Victoria-González B, Monsalve-Martín C, Duque-Heras JM, Juanes-Hernández MJ, Saura-Contí J, Soto-Sáez JL, Román-Moraleda C, Ruiz-Arias C, Martín-Mora B, Escolano-García R, Cantero-Bengoechea JS, García-López E, López-Pelegrín A, Padilla-Martin E, Martínez-Rodríguez M, Casillas-Martín J, Jerez-Vázquez J, Barrientos-Gómez L; Spanish Back Pain Research Network. Effectiveness of the Godelieve Denys- Struyf (GDS) Method in People With Low Back Pain: Cluster Randomized Controlled Trial. Phys Ther 2015; 95(3):319-336.
43. Zadro J, O'Keeffe M, Maher C. Do physical therapists follow evidence-based guidelines when managing musculoskeletal conditions? Systematic review. BMJ Open 2019; 9:e032329.

44. Seco J, Kovacs FM, Urrutia G. The efficacy, safety, effectiveness, and cost-effectiveness of ultrasound and shock wave therapies for low back pain: A systematic review. Spine J 2011; 11(10):966-977.

45. Urrútia G, Kovacs F, Nishishinya MB, Olabe J. Percutaneous thermocoagulation intradiscal techniques for discogenic low back pain. Spine (Phila Pa 1976) 2007; 32(10):1146-1154.

46. Traeger AC, Moynihan RN, Maher CG. Wise choices: making physiotherapy care more valuable. J Physiother 2017; 63(2):63-65.

47. Gérvas J, Fernández MP. Sobrediagnóstico, un problema clínico, ético y social. FMC Form Medica Contin en Aten Primaria 2014; 21(3):137-142.

48. Gérvas J, Pérez Fernández M. Aventuras y desventuras de los navegantes solitarios en el Mar de la Incertidumbre. Atención Primaria 2005; 35(2):95-98.

49. Heath I. Role of fear in overdiagnosis and overtreatment-an essay by Iona Heath. BMJ 2014; 349(October):22-25.

50. Atul Gawande. Overkill. The New Yorker. [accesado en 2019 Jun 8]. Disponíble en: https://www.newyorker. com/magazine/2015/05/11/overkill-atul-gawande

51. Coll-Benejam T, Bravo-Toledo R, Marcos-Calvo MP, Astier-Peña MP. Impact of overdiagnosis and overtreatment on the patient, the health system and society. Aten Primaria 2018; 50:86-95.
Artículo presentado en 13/10/2019

Aprobado en 23/10/2019

Version final presentada en 25/10/2019 